\title{
Antropometria e desempenho motor de atletas de Futebol de 7
}

Anthropometric and Motor Performance of Cerebral Palsy Football Athletes

\section{Fernando Rosch de Faria}

Laboratório de Avaliação Física no Exercício e Esporte Adaptados da Faculdade de Educação Física, Universidade Estadual de Campinas, Brasil

nando.rosch@yahoo.com.br

\section{Mariane Borges}

Laboratório de Avaliação Física no Exercício e Esporte Adaptados da Faculdade de Educação Física, Universidade Estadual de Campinas, Brasil

mariane9@yahoo.com.br

\section{Jéssica Reis Buratti}

Laboratório de Avaliação Física no Exercício e Esporte Adaptados da Faculdade de Educação Física, Universidade Estadual de Campinas, Brasil

jerburatti@gmail.com

\section{Claudio Diebl Nogueira}

Faculdade de Educação Física, Universidade Castelo Branco, Brasil

cdnogueira@hotmail.com

\section{Ivaldo Brandão Vieira}

Universidade Católica San Antonio de Murcia. Comitê Paralímpico Brasileiro., Brasil

ivaldobrandao@cpb.org.br

\section{Priscila Samora Godoy}

Educação Física pela Faculdade de Educação Física, Universidade Estadual de Campinas, Brasil prisamoragodoy@yahoo.com.br

\section{José Irineu Gorla}

Departamento de Estudos da Atividade Física Adaptada, Coordenador do Laboratório de Avaliação Física no Exercício e Esporte Adaptados da Faculdade de Educação Física, Universidade Estadual de Campinas, Brasil gorla@fef.unicamp.br

\section{Resumo:}

Introdução: $\mathrm{O}$ futebol de 7 é um esporte intermitente no qual a capacidade de força é evidenciada, tornando a avaliação desta e outras capacidades essenciais para o alcance do desempenho esportivo. Objetivo: $\mathrm{O}$ objetivo deste estudo foi identificar o perfil antropométrico e do desempenho motor, por classe funcional e posição de atletas da seleção brasileira de futebol de 7 . Metodologia: A amostra foi composta por 16 atletas. Na avaliação antropométrica foi medido: MC, estatura, IMC, pregas cutâneas e circunferência abdominal. Para o desempenho motor foram utilizados: saltos verticais, agilidade $10 \times 5$ e RAST test. Resultados:

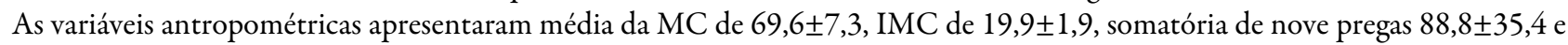

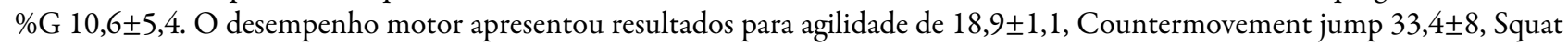


jump 31,6 $\pm 7,6$ e potência média 6,3 $\pm 1,5$. Conclusão: Os resultados se apresentaram adequados para atletas de alto rendimento e o desempenho motor sofreu influências da classe funcional.

Palavras-Chave: Avaliação, Antropometria, Desempenho motor, Futebol de 7, Paralisia Cerebral.

\section{Abstract:}

Football 7 is an intermittent sport, which requires strength capacity, making its evaluation and the evaluation of other capacities essential for the achievement of the sport performance. The aim of study was to identify the anthropometric profile and the motor performance of the athletes from the Brazilian National Cerebral Palsy Football Team. The sample was composed of 16 athletes. The Anthropometric assessment was made to weight, height, BMI, skinfolds and waist circumference. The motor performance was measured through vertical jump tests, $10 \times 5$ meters agility test and RAST test. Results: The anthropometric variables showed that the body mass $(\mathrm{Kg})$ was $69.6 \pm 7.3$, BMI was $19.9 \pm 1.9$, sum of 9 skinfolds $88.3 \pm 34.8$ and body fat percentage was $10.6 \pm 5.4$. The motor performance indicated that the agility test was $18.9 \pm 1.1$, the countermovement jump was $33.4 \pm 8$, the squat jump was 31.6 \pm 7.6 and average power was $6.3 \pm 1.5$. The results revealed to be consistent with high-performance athletes and also show that the functional class influenced their motor performance.

KEYWORDS: Evaluation, Anthropometry, Motor Performance, Football Seven-a-side, Cerebral Palsy.

\section{INTRODUÇÃO}

A Paralisia Cerebral (PC), ou encefalopatia crônica da infância, pode ser definida como um distúrbio não progressivo da motricidade causado por uma lesão no sistema nervoso central. Além disso, dependendo da região cerebral e extensão do acometimento, pode ocorrer uma disfunção no tônus muscular com intensidades variadas, influenciando na aptidão física e no desempenho destes sujeitos (Mattos, 2013).

Os indivíduos acometidos com tal deficiência podem praticar diversos esportes paralímpicos, dentre eles o futebol de 7 que é regido por regras similares ao futebol convencional adotando algumas adaptações em virtude das características desta população. Dentre as adaptações podemos destacar o número jogadores que é de sete, as dimensões máximas do campo que são de $75 \mathrm{~m}$ x $55 \mathrm{~m}$, a baliza de gol que é de $5 \mathrm{~m}$ x $2 \mathrm{~m}$ e a duração da partida que é disputada em dois períodos de 30 minutos (IFCPF, 2015b).

Além das adaptações descritas acima, para poderem competir os atletas são classificados de acordo com a deficiência e sua funcionalidade perante a modalidade, sendo lhes atribuídos 4 classes funcionais (CF) (CF5, CF6, CF7 e CF8) (Cruz, 2012). Segundo IFCPF (2015a) a CF 5 é concedida a atletas que apresentem diplegia, dupla hemiplegia e distonia, com moderado envolvimento espásticos, possuem membros inferiores mais afetados que os superiores e apresentam comprometimento no equilíbrio e passadas curtas; os atletas da CF 6 podem apresentar atetose, ataxia, distonia ou uma paralisia mista, as quais possuem características: moderado envolvimento nos quatro membros, dificuldade na marcha, descoordenação de movimentos, comprometimento no equilíbrio, dificuldade para mudanças de direção e paradas com e sem a bola, dificuldade de execução de movimentos explosivos e saltos, e hesitação na aceleração e aumento de impacto na desaceleração.

A CF 7 é atribuída aos hemiplégicos, que apresentem moderada espasticidade, com visível dificuldade de movimentação do lado acometido, boa habilidade funcional no lado do corpo preservado, comprometimento no equilíbrio, controle do joelho e quadril também são afetados pela espasticidade e possível perda de amplitude de movimento devido à contratura, aumento do tônus muscular nos membros superiores durante a corrida, devido a assimetria possuem força reduzida e dificuldade na execução de saltos. Por fim, os atletas que se enquadram na CF 8, são aqueles que podem apresentar monoplegia, além de todas as características acima citadas, porém com acometimentos mínimos (IFCPF, 2015a).

Com relação aos aspectos metabólicos inerentes à modalidade, o futebol de 7 apresenta similaridade ao futebol convencional, sendo que o metabolismo aeróbio é predominante, contudo o anaeróbio é determinante (Stolen, Chamari, Castagna, \& Wisløff, 2005) vide sua caracterização como um exercício intermitente na qual se alternam períodos de alta e baixa intensidade (Denadai, 2002). Tal característica 
intermitente está expressa em atividades como saltos, marcação, dribles, deslocamentos em velocidade e mudanças de direção (Stolen et al., 2005) evidenciando assim a manifestação da capacidade de força através de suas derivadas: potência, agilidade e velocidade.

A avaliação e monitoramento dessas capacidades, assim como das dimensões antropométricas que desempenham papel determinante (algumas vezes principal) no potencial de sucesso no esporte escolhido (Norton \& Olds, 2005), é fundamental para se alcançar o desempenho desejado, pois é através da avaliação que planejamentos prévios para trabalhos subsequentes são realizados, sendo que, é através de tais avaliações que metas são estabelecidas (Costa e Silva, Gorla, \& Costa, 2010). No entanto, não existem parâmetros que possam indicar quais são os perfis, seja antropométrico ou de desempenho motor que atletas de futebol de 7 apresentam.

Mediante ao exposto, delineou-se este estudo a fim de identificar o perfil antropométrico e do desempenho motor, por classe funcional e função em campo, de atletas da seleção brasileira de futebol de 7 .

\section{Materiais e Métodos}

Este estudo caracteriza-se como caráter descritivo com delineamento transversal, o mesmo foi submetido e aprovado pelo Comitê de Ética em Pesquisa da Faculdade de Ciências Médicas da Unicamp (Parecer CEP 709.135). Os participantes assinaram o Termo de Consentimento Livre e Esclarecido previamente à coleta dos dados.

\section{Amostra}

A amostra foi composta por 16 atletas com paralisia cerebral do sexo masculino na faixa etária de 26,3 ( $\pm 6,7)$ anos, integrantes da Seleção Brasileira de Futebol de 7 (Tabela 1).

TABELA 1

Características dos atletas pertencentes a Seleção Brasileira de Futebol de 7.

\begin{tabular}{|r|l|l|l|l|}
\hline Atleta & C.F & ID & Função & Tipo/Deficiência \\
\hline$J 1$ & 7 & 18 & Ala & Hemiparesia \\
\hline$J 2$ & 7 & 18 & Ala & Hemiparesia \\
\hline$J 3$ & 8 & 19 & Ala & Monoplegia \\
\hline$J 4$ & 6 & 23 & Ala & Atetose \\
\hline$J 5$ & 7 & 24 & Ala & Hemiparesia \\
\hline$J 6$ & 8 & 21 & Meia & Monoplegia \\
\hline$J 7$ & 7 & 24 & Meia & Hemiparesia \\
\hline$J 8$ & 8 & 27 & Meia & Monoplegia \\
\hline$J 9$ & 7 & 22 & Atacante & Hemiparesia \\
\hline$J 10$ & 7 & 26 & Atacante & Hemiparesia \\
\hline$J 11$ & 6 & 36 & Atacante & Atetose \\
\hline$J 12$ & 7 & 27 & Zagueiro & Hemiparesia \\
\hline$J 13$ & 7 & 30 & Zagueiro & Hemiparesia \\
\hline$J 14$ & 7 & 30 & Goleiro & Hemiparesia \\
\hline$J 15$ & 7 & 36 & Goleiro & Hemiparesia \\
\hline$J 16$ & 6 & 40 & Goleiro & Atetose \\
\hline & & & & \\
\hline
\end{tabular}




\section{Antropometria}

As variáveis antropométricas massa corporal e estatura foram mensuradas através de uma balança da marca Filizola com escala de leitura em gramas e um estadiômetro de madeira com escala de leitura em milímetros.

O Índice de Massa Corporal - IMC foi obtido pela divisão da massa $(\mathrm{Kg})$ pela estatura elevada ao quadrado $\left(\mathrm{m}^{2}\right)$. Para a mensuração da espessura de pregas cutâneas foi utilizado o adipômetro da marca Harpenden , sendo aferidas as pregas: Tríceps, Subescapular, Bíceps, Torácica, Axilar, Supra ilíaca, Abdominal, Coxa e Panturrilha, de acordo com o protocolo de Guedes e Guedes (2006). Todas as medidas foram realizadas consecutivamente por três vezes em um mesmo local, considerando-se o valor mediano como medida representativa.

Para a estimativa da densidade corporal foi utilizado o protocolo de Jackson e Pollock (Jackson \& Pollock, 1978) $\left[\mathrm{D}=1,112-0,00043499(\mathrm{x} 1)+0,00000055(\mathrm{x} 1)^{2}-0,00028826(\mathrm{x} 3)\right]$ o qual utiliza as pregas Torácica, Axilar, Tricipital, Subescapular, Abdominal, Supra ilíaca e Coxa para o cálculo da densidade corporal. A fim de aferir o percentual de gordura corporal (\%G) utilizou-se a equação de Siri (1961): \%G = (4.95/Densidade $-4.50)^{*} 100$.

Também foi mensurada a circunferência abdominal através de uma fita antropométrica com escala de leitura em milímetros. Tal medida foi selecionada por ser um dos principais preditores de composição corporal e de risco cardiovascular para indivíduos com e sem deficiência (Ravensbergen, Lear, \& Claydon, 2014).

\section{Testes Motores}

\section{Rast Test (Running-based Anaerobic Sprint Test)}

O Runnins Anaerobic Sprint Test (RAST) tem por objetivo avaliar a potência e a capacidade anaeróbia, mensurando os valores de potência máxima (Pmáx), Potência média e Potência mínima, além do Índice de Fadiga (IF) na corrida (Zacharogiannis, Paradisis, \& Tziortzis, 2004). O teste consiste na realização de 6 esforços em um percurso linear de 35 metros em velocidades máximas, alternados com 10 segundos de recuperação passiva; e são computados os tempos em segundos de cada esforço realizado. Para a realização do teste e captura da velocidade, foram utilizadas duas fotocélulas eletrônicas Speed Test 4.0 (CEFISE).

\section{Teste de Agilidade $10 X 5$ metros}

Proposto por Guedes e Guedes (2006), o teste tem por objetivo avaliar o componente motor associado à velocidade de deslocamento, mediante corridas com mudanças de direção por percurso de 50 metros, iniciando-se da posição parada. O solo deve ser marcado com duas linhas paralelas, com 5 metros de distância, nas extremidades devem ser indicadas por cones. $\mathrm{O}$ avaliado deve correr o mais rápido possível em direção a outra linha, transpondo-a e retornando a linha de chegada. Ambas as linhas devem ser transpostas com os dois pés. Computa-se o tempo com definição em centésimos de segundo, despendido pelo avaliado para completar os cinco ciclos (permitida uma única tentativa). 


\section{Saltos Verticais}

O teste de saltos verticais tem por objetivo estimar a força e potência da musculatura extensora das pernas. O teste consiste na realização dos saltos: Squat Jump (SJ) e Countermovement Jump (CMJ) (Bosco, Luhtanen, \& Komi, 1983). Foram realizados 3 saltos com 10 segundos de recuperação passiva entre cada salto. Para a performance do SJ, na posição inicial os atletas deveriam estar com uma flexão de joelho a $90^{\circ}$ e ambas as mãos no quadril, durante a fase de voo os membros inferiores deveriam ser mantidos em extensão até o contato com o solo; e no CMJ os atletas foram instruídos a se posicionarem com os membros inferiores em extensão e no momento do salto realizar a flexão de joelho em no máximo $90^{\circ} \mathrm{com}$ ambas as mãos no quadril, durante a fase de voo os membros inferiores deveriam ser mantidos em extensão até o contato com o solo. Os saltos verticais foram realizados através do equipamento Jump System Pro, Tapete de contato CEFISE. Computador com o software Jump System Pro.

\section{Análise Estatistica}

Os dados foram apresentados por meio da estatística descritiva através de média e desvio padrão. A análise de normalidade foi realizada através do teste Shapiro-Wilk. Para as análises de diferença entre as classes funcionais, função em campo para cada variável e entre as classes funcionais para as diferentes voltas no teste de potência (rast test) foi utilizada o teste de Kruskal-Wallis. Os dados foram analisados através do pacote estatístico R-Plus versão 2.15.0 2012, para Windows (Viena, Áustria) e o pacote R-Studio (Boston, EUA). O nível de significância adotado foi de $\mathrm{p} \leq 0,05$.

\section{Resultados}

Os resultados apontam que não houve diferença significativas nas variáveis antropométricas e da composição corporal entre os grupos de acordo com suas classes funcionais (Tabela 2).

TABELA 2

Média e desvio padrão para características antropométricas e da composição corporal por classe funcional.

\begin{tabular}{lllll}
\hline Variáveis & CF 6 $(\mathrm{n}=4)$ & CF 7 (n=9) & CF 8 (n=3) & Grupo \\
\hline Idade (anos) & $33( \pm 8,9)$ & $25,5( \pm 5,6)$ & $22,3( \pm 4,2)$ & $26,3( \pm 6,7)$ \\
Est (m) & $1,74( \pm 0,1)$ & $1,76( \pm 0,05)$ & $1,73( \pm 0,1)$ & $1,75( \pm 0,1)$ \\
MC (Kg) & $70,5( \pm 7,4)$ & $70,5( \pm 8,4)$ & $65,6( \pm 1,9)$ & $69,6( \pm 7,3)$ \\
IMC (Kg/m2) & $20,3( \pm 2,5)$ & $20,1( \pm 2,1)$ & $19( \pm 0,6)$ & $19,9( \pm 1,9)$ \\
\%G & $13,2( \pm 6,6)$ & $11,2( \pm 5,5)$ & $6,3( \pm 0,2)$ & $10,6( \pm 5,4)$ \\
MG (Kg) & $9,6( \pm 5,4)$ & $8,2( \pm 5)$ & $4,1( \pm 0,3)$ & $7,7( \pm 4,7)$ \\
MM (Kg) & $60,9( \pm 2,2)$ & $62,3( \pm 5,1)$ & $61,5( \pm 1,6)$ & $61,9( \pm 4,1)$ \\
Soma 9(mm) & $101( \pm 40,9)$ & $93,6( \pm 37,1)$ & $60,7( \pm 5,4)$ & $88,8( \pm 35,4)$ \\
C. Abd (cm) & $82,5( \pm 5,9)$ & $75( \pm 15,8)$ & $75,2( \pm 2,6)$ & $76,4( \pm 12,8)$ \\
\hline
\end{tabular}


Legenda: Est=estatura; $\mathrm{MC}=$ massa corporal; $\mathrm{IMC}=$ índice de massa corporal; $\% \mathrm{G}=$
percentual de gordura corporal; $\mathrm{MG}=$ massa gorda corporal; $\mathrm{MM}=$ massa magra corporal;
Soma $9=$ somatória das nove pregas cutâneas aferidas; $\mathrm{C} . \mathrm{Abd}=$ circunferência abdominal.

Como constatado, atletas da CF8 apresentaram resultados superiores aos das demais classes funcionais em todas as variáveis estudadas referente ao desempenho motor. Sendo demonstradas diferenças significativas $(\mathrm{p} \leq 0,05)$ para as variáveis agilidade e potência média (Tabela 3).

TABELA 3

Média e desvio padrão para o desempenho motor por classe funcional.

\begin{tabular}{lllll}
\hline Variáveis & CF 6 $(\mathrm{n}=4)$ & CF 7 $(\mathrm{n}=9)$ & CF 8 $(\mathrm{n}=3)$ & Grupo \\
\hline $\mathrm{Ag}(\mathrm{seg})$ & $20,1( \pm 1)$ & $18,9( \pm 0,9)$ & $17,8( \pm 0,6) 1$ & $18,9( \pm 1,1)$ \\
$\mathrm{CMJ}$ & $27,8( \pm 7,7)$ & $34,2( \pm 6,9)$ & $36,3( \pm 12)$ & $33,4( \pm 8)$ \\
SJ & $26,6( \pm 7,5)$ & $32( \pm 6,9)$ & $35,5( \pm 10)$ & $31,6( \pm 7,6)$ \\
Pot Med (W/Kg) & $4,8( \pm 0,9)$ & $6,1( \pm 1,2)$ & $8,3( \pm 0,7) \mathbf{1 2}$ & $6,3( \pm 1,5)$ \\
IF (W/Seg) & $6( \pm 2,1)$ & $7,8( \pm 3,2)$ & $8,3( \pm 1,9)$ & $7,5( \pm 2,8)$ \\
\hline
\end{tabular}

Legenda: CF - Classe funcional; Ag - Agilidade; CMJ - Countermovment jump; SJ - Squat jump; CMJB - Countermovment jump com utilização dos braços; Pot Med - Potência Média; IF - Índice de fadiga. 1Diferença significativa $(\mathrm{p} \leq 0,05)$ com a CF6. 2Diferença significativa $(\mathrm{p} \leq 0,05)$ entre a CF7.

Em relação ao Rast test (figura 1), as médias de potência dos atletas CF 8 foi significativamente $(\mathrm{p} \leq 0,05)$ superior nos sprints de 2 a 6 e 3 a 6 , para com a CF 6 e CF 7, respectivamente. Entre as classes 6 e 7 , houve diferença significativa nos sprints 4 e 5 . Ainda mais, observa-se que atletas CF 8 e CF 7 obtiveram maior sprint na segunda volta, enquanto os CF 6 na primeira volta. 
FIGURA 1

Potência estimada em cada Sprint por classe funcional.

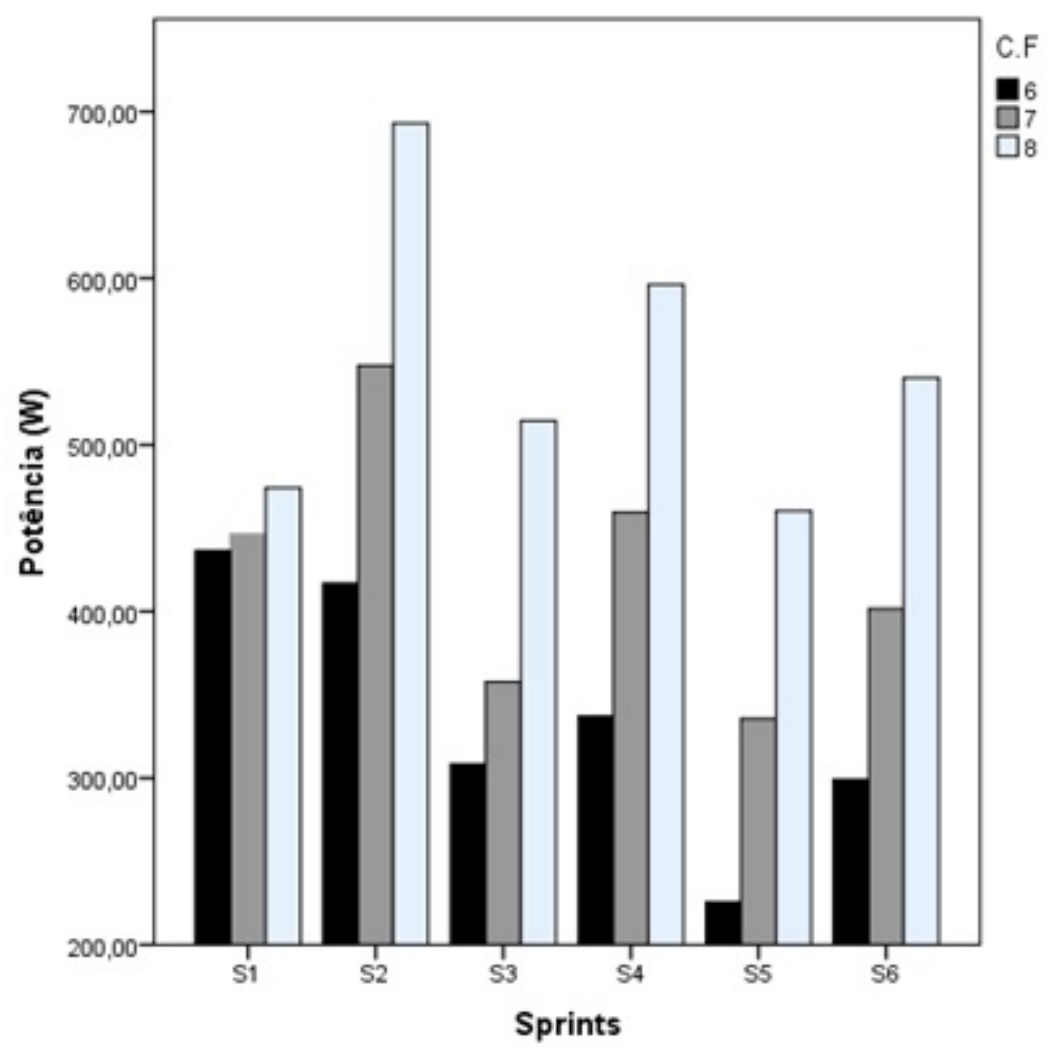

Concernente as informações antropométricas e de desempenho motor descritas por função de jogo (Tabela 4), pode-se observar que houve diferença significativa $(\mathrm{p} \leq 0,05)$ em: Idade (Goleiros com Meias e Alas); Massa corporal (Goleiros com Alas e Meias, Atacantes com Alas e Meias); Massa Gorda e Soma de 9 pregas (Goleiros com Alas e Meias, Meias com Atacantes) e Potência Media (Goleiros com Alas, Meias e Atacantes, e Meias com Atacantes). 
TABELA 4

Características antropométricas e de desempenho motor de acordo com a função de jogo.

\begin{tabular}{|r|l|l|l|l|l|}
\hline Variáveis & Alas $(\boldsymbol{n}=\mathbf{5})$ & Goleiros $(\boldsymbol{n}=\mathbf{3})$ & Meias $(\boldsymbol{n}=\mathbf{3})$ & Atacantes $(\boldsymbol{n}=\mathbf{3})$ & Zagueiros $(\boldsymbol{n}=\mathbf{2})$ \\
\hline Idade & $20,4( \pm 2,9)^{2}$ & $35,3( \pm 5)^{13}$ & $24( \pm 3)_{n}^{2}$ & $28( \pm 7,2)$ & $28,5( \pm 2,1)$ \\
\hline Est $(\mathrm{m})$ & $1,78( \pm 0,03)$ & $1,80( \pm 0,01)$ & $1,70( \pm 0,02)$ & $1,70( \pm 0,1)$ & $1,70( \pm 0,1)$ \\
\hline$M C(\mathrm{~kg})$ & $66,4( \pm 2,6)^{24}$ & $80,1( \pm 6,4)^{13}$ & $62,6( \pm 4,1)^{24}$ & $72,1( \pm 3,4)^{13}$ & $68,8( \pm 9,5)$ \\
\hline$I M C \mathrm{~kg} / \mathrm{m}^{2}$ & $18,7( \pm 0,8)$ & $22,4( \pm 1,8)$ & $18,6( \pm 1,2)$ & $20,7( \pm 1,7)$ & $20,1( \pm 2,1)$ \\
\hline$\% G$ & $8,0( \pm 2,5)$ & $18,8( \pm 4,3)$ & $6,2( \pm 0,1)$ & $11,3( \pm 6,6)$ & $10,7( \pm 1,0)$ \\
\hline$M G(\mathrm{~kg})$ & $5,3( \pm 1,8)_{n}^{2}$ & $15,1( \pm 4)^{13}$ & $3,9( \pm 0,2)^{24}$ & $8,3( \pm 5,2)$ & $7,3( \pm 0,3)$ \\
\hline$M M(\mathrm{~kg})$ & $61( \pm 1,7)$ & $65( \pm 4,9)$ & $58,7( \pm 3,9)$ & $63,8( \pm 1,9)$ & $61,5( \pm 9,1)$ \\
\hline Soma $9(\mathrm{~mm})$ & $76,1( \pm 19)_{n}^{2}$ & $139,1( \pm 35,6)^{13}$ & $58,4( \pm 3,3)^{24}$ & $91,6( \pm 42,1)$ & $86,5( \pm 7,8)$ \\
\hline$C . A b d(\mathrm{~cm})$ & $75,5( \pm 2,2)$ & $88,7( \pm 4,2)$ & $74,5( \pm 3,5)$ & $81,3( \pm 5,5)$ & $55,8( \pm 30,8)$ \\
\hline$A g(\mathrm{seg})$ & $18,6( \pm 0,6)$ & $19,3( \pm 1,2)$ & $17,8( \pm 0,6)$ & $20( \pm 1,5)$ & $19( \pm 0,4)$ \\
\hline$C M J(\mathrm{~cm})$ & $34,7( \pm 7,5)$ & $25( \pm 4,9)$ & $40,9( \pm 4,0)$ & $28,9( \pm 9)$, & $38,1( \pm 0,8)$ \\
\hline$S J$ & $31,1( \pm 6,3)$ & $25,5( \pm 5,4)$ & $39,1( \pm 3,8)$ & $29,1( \pm 11,8)$ & $33,6( \pm 5,1)$ \\
\hline Pot $\mathrm{Med}(\mathrm{W} / \mathrm{Kg})$ & $6,8( \pm 0,9)_{n}^{2}$ & $4,1( \pm 0,4)^{134}$ & $8,2( \pm 0,8)^{24}$ & $5,6( \pm 0,6)^{23}$ & $6,5( \pm 0,2)$ \\
\hline$I F(\mathrm{~W} / \mathrm{Seg})$ & $7,4( \pm 2,1)$ & $4,9( \pm 1,7)$ & $8,5( \pm 1,9)$ & $7,2( \pm 4,3)$ & $10,8( \pm 1,4)$ \\
\hline
\end{tabular}

Legenda: Est - estatura; MC - massa corporal; IMC - índice de massa corporal; \%G - percentual de gordura corporal; MG - massa gorda corporal; MM - massa magra corporal; Soma 9 - somatória das nove pregas cutâneas aferidas; C.Abd - circunferência abdominal; Ag - Agilidade; CMJ - Countermovment jump; SJ - Squat jump; CMJB - Countermovment jump com utilização dos braços; Pot Med - Potência Média; IF - Índice de fadiga; 1 Diferença significativa com Alas; 2 Diferença significativa com Goleiros; 3 Diferença significativa com Meias; 4 Diferença significativa com Atacantes.

\section{DISCUSSÃO}

Objetivou-se através deste estudo identificar o perfil antropométrico e do desempenho motor, por classe funcional e função em campo, de atletas da seleção brasileira de futebol de 7.

No que tange a variável IMC o grupo estudado é considerado como peso normal (WHO, 2000) e comparado ao IMC de jogadores da seleção irlandesa de futebol de sete 24,4 2,2 (Kloyiam, Breen, Jakeman, Conway, \& Hutzler, 2011) os mesmos apresentam melhores resultados $(19,9 \pm 1,9)$. No entanto, sabe-se que o IMC possui grandes limitações principalmente pelo fato de não considerar massa gorda de massa magra, o que acaba por prejudicar sua estimação como indicador de sobrepeso e obesidade na população em geral.

Bons resultados também podem ser constatados quanto a variável percentual de gordura corporal em relação ao estudo de Kloyam et al., (2011) os quais apresentaram valor médio de 22 \$5,8, e também a valores de atletas de futebol profissional sem deficiência que varia de 10 à 12\% (Do Prado et al., 2006; Osiecki, Glir, Fornaziero, Cunha, \& Dourado, 2007). Através destes resultados pressupõe-se que, os atletas alvos deste estudo, encontram-se dentro de um perfil antropométrico adequado para atletas de alto rendimento.

No que concerne ao desempenho motor, a potência de membros inferiores verificada pelos saltos verticais, observamos que a altura no salto CMJ e SJ dos atletas desse estudo foram superiores aos dos estudos de Yanci et al., (2014) e Yanci et al., (2016) os quais avaliaram jogadores pertencentes a Seleção Espanhola de 


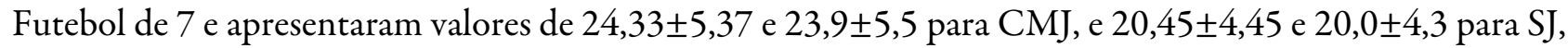
respectivamente.

Ainda referente a potência de membros inferiores, porém verificada através do Rast test, atletas de CF 8 obtiveram melhores resultados em potência média que atletas CF 6 e CF 7 (8,3 $\pm 0,7,4,8 \pm 0,9$ e $6,1 \pm 1,2$, respectivamente). Resultado este superior à média do grupo $(6,3 \pm 1,5)$ e ao de Souza et al., (2012) que obtiveram $6 \pm 1$ em jogadores sem deficiência, o que suporta nossa premissa de tais resultados superiores com as demais classes estejam relacionados ao fato de atletas de CF 8 apresentarem acometimentos mínimos da PC. Outro resultado similar ao de Souza et al., (2012) trata-se de os CF 8 terem como melhor volta o sprint $2(\mathrm{p}=0,024)$, estatisticamente superior as demais classes.

Com relação a agilidade, constatou-se que os indivíduos da CF8 apresentaram resultados $(17,8 \pm 0,5)$ significativamente melhores que os da CF6 $(19,9 \pm 0,9)$. As características das classes pode ser um fator influenciador, visto que atletas CF 6 detém de equilíbrio e capacidade de mudança de direção prejudicada mediante sequelas da PC, enquanto os CF8 possuem funções próximo do normal (IFCPF, 2015a).

Não houve diferença nas variáveis da composição corporal em relação as $\mathrm{CF}$, porém verificou-se diferenças ao serem realizadas análises de acordo com as funções exercidas em campo. Observou-se que a massa gorda corporal de Alas $(66,4 \pm 2,6)$ e Meias $(62,6 \pm 4,1)$ foram significativamente $(\mathrm{p} \leq 0,05)$ inferiores aos resultados apresentados pelos goleiros $(80,1 \pm 6,4)$, corroborando com os estudos de Lima, Martins, Liberali \& Navarro (2009) e Do Prado et al. (2006). O fato de goleiros apresentarem maior massa corporal que os demais atletas pode ser explicado pelos padrões táticos da função, que implica em menor distância total percorrida bem como o tipo e a intensidade das ações realizadas (Lima et al., 2009).

Assim como para as variáveis da composição corporal, as variáveis do desempenho motor também foram analisadas de acordo com a função exercida em campo por cada jogador, das quais foram encontradas diferenças significativas dos Goleiros com Meias, Atacantes e Alas ( $p=0,002 ; 0,022 ; 0,005)$, e dos Meias com Atacantes $(\mathrm{p}=0,015)$ na potência média. A força máxima é essencial para a performance da potência (Ronnestad, Kvamme, Sunde, \& Raastad, 2008), e visto que pessoas com PC possuem um déficit de força muscular (Damiano \& Abel, 1998; Krigger, 2006; Silva \& Andrade, 2002), acredita-se que o grau de acometimento nos membros inferiores nos Goleiros possam ter contribuído para resultados. No caso dos Meias atingirem melhores resultados que os atacantes, o mesmo pode ser levado em consideração, visto que os Meias, neste estudo, possuem atletas com menor grau de acometimento (Tabela 1).

Considerando que a convocação da Seleção Brasileira de Futebol de 7 como em outros esportes são selecionados os melhores atletas dispostos em suas posições e classes funcionais como requerido pelas regras da modalidade justifica o tamanho da amostra. O perfil antropométrico e o desempenho motor, podem fornecer parâmetros de comparação para futuras pesquisas com outras equipes da população em questão.

Ressalta-se a escassez de pesquisas na modalidade, o que limita o potencial de comparação com este estudo, levando a contrastar os resultados com atletas de futebol sem deficiência, fato este que reforça a importância de desenvolvimento desta pesquisa.

\section{CONCLUSÃO}

Nota-se através dos resultados que houve diferença significativa entre as classes nas variáveis do desempenho motor: agilidade e potência média, e entre as posições nas variáveis antropométricas: idade, massa corporal, massa gorda, soma de 9 pregas, e potência média no desempenho motor.

Observou-se que os atletas CF 6 apresentaram resultados significativamente inferiores nas variáveis de desempenho motor (agilidade e potência média) em relação as outras $\mathrm{CF}$, como esperado os atletas $\mathrm{CF}$ 8 obtiveram resultados significativamente superiores nas variáveis do desempenho motor, e em relação as posições de jogo os goleiros obtiveram resultados inferiores em relação as demais posições. 
Através deste estudo podemos concluir que o perfil da composição corporal de atletas de futebol de 7 pode ser considerado como adequado para atletas de alto rendimento, e que o desempenho motor desses atletas sofre influências da classe funcional, sugerindo que atletas com maior grau de acometimento apresentarão resultados inferiores.

\section{Agradecimentos}

Associação Nacional de desporto para deficientes - ande laboratório integrado de ensino, pesquisa e extensão - labfef.

\section{REFERÊNCIAS}

Bosco, C., Luhtanen, P., \& Komi, P. V. (1983). A simple method for measurement of mechanical power in jumping. Eur J Appl Physiol Occup Physiol, 50(2), 273-282.

Costa e Silva, A. A., Gorla, J. I., \& Costa, L. T. (2010). Avaliação Motora em Esporte Adaptado. In A. F. Machado (Org.), Manual de Avaliação Física (1o ed, p. 227-247). São Paulo: Ícone.

Cruz, P. (2012). Futebol de Sete. In M. T. de; Mello \& C. Winckler (Orgs.), Esporte Paralímpico (1a, p. 1-256). São Paulo: Atheneu.

Damiano, D. L., \& Abel, M. F. (1998). Functional outcomes of strength training in spastic cerebral palsy. Archives of Physical Medicine and Rehabilitation, 79(2), 119-125. http://doi.org/10.1016/S0003-9993(98)90287-8

Denadai, B. S. (2002). Determinação do limiar anaeróbio em jogadores de futebol com paralisia cerebral e nadadores participantes da paraolimpíada de Sidney 2000. Revista Brasileira de Medicina do Esporte, 8(3), 117-121. http:// doi.org/10.1590/S1517-86922002000300009

Do Prado, W. L., Botero, J. P., Guerra, R. L. F., Rodrigues, C. L., Cuvello, L. C., \& Dâmaso, A. R. (2006). Perfil antropométrico e ingestão de macronutrientes em atletas profissionais Brasileiros de futebol, de acordo com suas posições. Revista Brasileira de Medicina do Esporte, 12(2), 61-65. http://doi.org/10.1590/ S1517-86922006000200001

Guedes, D. P., \& Guedes, J. E. R. P. (2006). Manualprático para avaliação em Educação Física (1o ed). Barueri: Manole.

IFCPF, I. F. of C. P. F. (2015a). Classification Rulebook. International Federation of Cerebral Palsy Football. Worcester, South Africa. Recuperado de http://www.ifcpf.com/static/upload/raw/d4e7103a-d030bef3-9eb6-51c529bd60e4/IFCPF+Classification+Rules+2015.pdf

IFCPF, I. F. of C. P. F. (2015b). CP Football Rules and Regulation. International Federation of Cerebral Palsy Football. Worcester, South Africa. Recuperado de http://www.ifcpf.com/static/upload/raw/1c9262a9-613f-55b0-799d-f3c8c408d692/IFCPF $+\mathrm{CP}+$ Football+Rules+and+Regulation+version+January+2015.pdf

Jackson, A., \& Pollock, M. (1978). 497 Generalized equations for predicting body density of men. British journal of nutrition, 40(1), 497-504.

Kloyiam, S., Breen, S., Jakeman, P., Conway, J., \& Hutzler, Y. (2011). Soccer-specific endurance and running economy in soccer players with cerebral palsy. Adapted Physical Activity Quarterly, 28(4), 354-367.

Krigger, K. W. (2006). Cerebral Palsy\#: An Overview. American Family Physician, 73(1), 90-100.

Lima, C. B. N., Martins, M. E. F., Liberali, R., \& Navarro, F. (2009). Estado nutricional de jogadores de futebol profissional. Revista Brasileira de Nutrição Esportiva, 3(18), 562-569. Recuperado de http://www.rbne.com.br/ index.php/rbne/article/viewFile/155/153

Mattos, E. (2013). Atividade física nos distúrbios neurológicos e musculares. In M. Greguol \& R. F. Costa (Orgs.), Atividade Fisica Adaptada (3o ed, p. 221-253). Barueri: Manole.

Norton, K., \& Olds, T. (2005). Atropométrica. Porto Alegre: Artmed. 
Osiecki, R., Glir, F. G., Fornaziero, A. M., Cunha, R. C., \& Dourado, A. C. (2007). Parâmetros antropométricos e fisiológicos de atletas profissionais de futebol. Revista da Educação Física/UEM, Maringa, 18(2), 177-182.

Ravensbergen, H. R. J. C., Lear, S. A., \& Claydon, V. E. (2014). Waist circumference is the best index for obesityrelated cardiovascular disease risk in individuals with spinal cord injury. Journal of neurotrauma, 31(3), 292300. http://doi.org/10.1089/neu.2013.3042

Ronnestad, B. R., Kvamme, N. H., Sunde, A., \& Raastad, T. (2008). Short-term effects of strength and plyometric training on sprint and jump performance in professional soccer players. Journal of strength and conditioning research, 22(3), 773-80. http://doi.org/10.1519/JSC.0b013e31816a5e86

Silva, A. C., \& Andrade, M. D. S. (2002). Avaliação isocinética em atletas paraolímpicos. Revista Brasileira de Medicina do Esporte, 8(3), 99-101. http://doi.org/10.1590/S1517-86922002000300006

Siri, W.E. (1961). Composition from fluid spaces and density: analisys of methods. In J. Brozek \& A. Henschel (Orgs.), Techniques for measuring body composition (Vol. 61, p. 223-244). Washington: National Academy of Science.

Souza, V. amorim F. A., Pires, F. de O., Silva, A. E. L., \& Bertuzzi, R. (2012). Relação entre o desempenho no RunningBased Anaerobic Sprint Test (RAST) e a altura do salto vertical, salto horizontal e agilidade em Futebolistas. Revista Acta Brasileira do Movimento Humano, 2(1), 34-42.

Stolen, T., Chamari, K., Castagna, C., \& Wisløff, U. (2005). Physiology of Soccer. Sports Medicine, 35(6), 501-536. http://doi.org/10.2165/00007256-200535060-00004

WHO. (2000). Obesity: preventing and managing the global epidemic: Report of a World Health Organization Consultation (WHO technical report series\#; 894). WHO Consultation on Obesity. Geneva, Switzerland.

Yanci, J., Castagna, C., Los Arcos, A., Santalla, A., Grande, I., Figueroa, J., \& Camara, J. (2016). Muscle strength and anaerobic performance in football players with cerebral palsy. Disability and Health Journal, 9, 313-319.

Yanci, J., Los Arcos, A., Grande, I., Santalla, A., Figueroa, J., Gil, E., \& Cámara, J. (2014). Capacidad de salto en futbolistas con parálisis cerebral. Revista Internacional de Medicina y Ciencias de la Actividad Fisica y del Deporte, 14(54), 199-211.

Zacharogiannis, E., Paradisis, G., \& Tziortzis, S. (2004). An evaluation of tests of anaerobic power and capacity. Medicine \& Science in Sports \& Exercise, 36(5), 116. 